\title{
A Medium-Throughput Analysis of Signaling Pathways Involved in Early Stages of Stem Cell Reprogramming
}

\author{
Ashley L. Fritz, ${ }^{1}$ Sunnie R. Mao, ${ }^{1}$ Mary G. West, ${ }^{2}$ David V. Schaffer ${ }^{1,3,4}$ \\ ${ }^{1}$ Department of Chemical and Biomolecular Engineering, University of California, \\ Berkeley, California 94720 \\ ${ }^{2}$ Berkeley Stem Cell Center, University of California, Berkeley, California 94720 \\ ${ }^{3}$ Department of Bioengineering, University of California at Berkeley, Berkeley, California \\ 94720 \\ ${ }^{4}$ Helen Wills Neuroscience Institute, University of California, Berkeley, California 94720; \\ telephone: 510643 5963; fax: 510642 4778; e-mail: schaffer@berkeley.edu
}

\begin{abstract}
The induction of pluripotency from adult cells has enormous potential in regenerative medicine. While initial efforts to study mechanisms and improve efficiency of induced pluripotent stem cell (iPSC) reprogramming focused on the direct roles of transcriptional regulators, increasing evidence indicates that cellular signal transduction pathways can modulate this process. Here, we present a mediumthroughput system to study the effect of signaling pathways on the early stages of reprogramming. We generated a set of lentiviral vectors encoding 38 genes that upregulate or downregulate major signal transduction pathways and quantified each signaling factor's effect on reprogramming. This approach confirmed the role of several factors previously implicated in reprogramming, as well as identified several GTPases-factors that to date have not been largely studied in reprogramming-that improve or hinder iPSC reprogramming. In addition, this methodology is useful in determining new targets for enhancing pluripotency reprogramming, lineage reprogramming, and/or cell differentiation.

Biotechnol. Bioeng. 2015;112: 209-219.

(C) 2014 Wiley Periodicals, Inc.

KEYWORDS: induced pluripotent stem cells; reprogramming; signal transduction
\end{abstract}

Author contributions: Ashley L. Fritz and David V. Schaffer designed the experiments. Ashley L. Fritz and Sunnie R. Mao performed the experiments. Ashley L. Fritz, Sunnie R. Mao, and Mary G. West analyzed the data. Ashley L. Fritz and Sunnie R. Mao prepared the figures. Ashley L. Fritz wrote the manuscript, with editorial feedback and revision with David V. Schaffer.

Conflict of interest: We declare no potential conflicts of interest relevant to this article. Correspondence to: D.V. Schaffer

Contract grant sponsor: National Science Foundation Graduate Fellowship Contract grant number: NIH R01 ES020903

Received 1 April 2014; Revision received 28 May 2014; Accepted 7 July 2014

Accepted manuscript online 26 July 2014;

Article first published online 2 September 2014 in Wiley Online Library

(http://onlinelibrary.wiley.com/doi/10.1002/bit.25336/abstract).

DOI 10.1002/bit.25336
Yamanaka's groundbreaking work found that expression of only four transcription factors-OCT4, SOX2, KLF4, and CMYC (OSKM)-was sufficient to revert differentiated adult cells to embryonic phenotypes (Takahashi and Yamanaka, 2006). The resulting induced pluripotent stem (iPS) cells, which have the capacity to form all three germ layers and subsequently all cell types in an adult organism, offer immense biomedical potential including the use of patientderived iPS cells for cell-replacement therapies or for in vitro models of human disease. However, further understanding of reprogramming mechanisms is necessary for this relatively new technology, particularly to develop safer and potentially more efficient reprogramming methodologies for downstream applications.

Signal transduction, the relay of a microenvironmental input into the interior of a cell, is mediated through proteinprotein and second messenger interactions and is a central regulator of nearly all facets of cell behavior. In many cases, these signaling pathways can modulate the activity of key transcription factors, including the Yamanaka factors and other factors involved in pluripotency, strongly suggesting that signaling may modulate reprogramming. For instance, leukemia inhibitor factor (LIF) and bone morphogenic protein 4 (BMP4) activate signaling pathways and downstream effectors such as STAT3, which in turn activates CMYC as well as Inhibitor of Differentiation (ID) proteins to maintain murine embryonic stem cell pluripotency (Boeuf et al., 1997; Cartwright et al., 2005; Matsuda et al., 1999; Niwa et al., 1998; Raz et al., 1999; Ying et al., 2003). To date several signaling pathways have indeed been implicated in the reprogramming process. For example, early work demonstrated that small molecule inhibitors of TGF $\beta$ signaling can replace SOX2 (Ichida et al., 2009; Maherali and Hochedlinger, 2009), BMP signaling is important in the early state mesenchymal to epithelial transition (Samavarchi-Tehrani 
et al., 2010), and exogenous WNT3A can create pluripotent colonies in the absence of exogenous C-MYC (Marson et al., 2008).

Guided by the candidate approach that originally led to the identification of the four canonical reprogramming factors (Takahashi and Yamanaka, 2006), we have systematically investigated upstream signal transducers that could modulate reprogramming. Specifically, we generated a set of lentiviral vectors encoding 38 constitutively-active, dominant-negative, or wild-type versions of signal transduction proteins to probe several intracellular signaling pathways. By measuring the effect of each on reprogramming in a mediumthroughput, quantitative manner, we both validated the methodology by comparison to previously published results as well as discovered new roles for GTPase-mediated signal transduction pathways in early stages of reprogramming. Additionally, we have created an accessible and quantitative system to study signaling pathways for downstream applications in cellular biology.

\section{Results}

To study the effect of signaling pathways on OSKM iPS cell reprogramming, we first inserted 38 genes encoding signal transduction proteins into the lentiviral vector $\mathrm{pHIV} \mathrm{IG} \mathrm{loxP,}$ a modified version of the self-inactivating vector $\mathrm{PHIV}$-EGFP (Welm et al., 2008) (Fig. 1A). The signaling factors (Table I) are representative of several pathways important in mammalian cell biology: calcium-modulated, cytoskeletal, G-protein receptor-mediated, hypoxia-mediated, JAK-STAT, canonical tyrosine kinase receptor-mediated, NFкB, Notch, Hedgehog, TGF $\beta$, and Wnt- $\beta$-catenin signaling pathways.

After separately packaging vectors encoding the signaling factors, viral titer was determined via GFP expression on either HEK293T or MEF cells as previously described (Peltier and Schaffer, 2014). Briefly, $72 \mathrm{~h}$ after infection, viral transgene expression was assayed with high-content imaging and analysis to measure GFP expression (Fig. 1B-C). GFP fluorescence levels within the MEF cells were lower than in
A

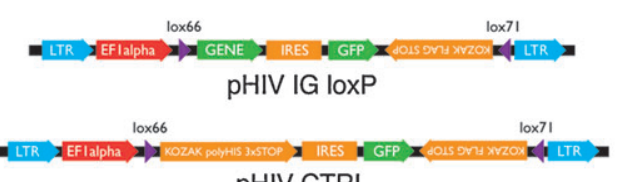

pHIV CTRL

C
B

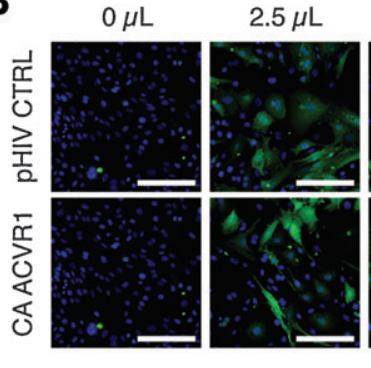

$5 \mu \mathrm{L}$

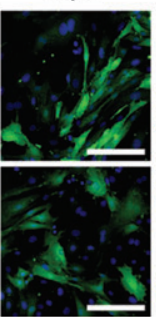

$10 \mu \mathrm{L}$

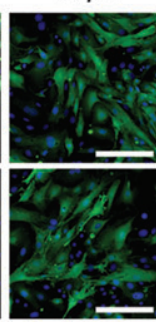

$20 \mu \mathrm{L}$

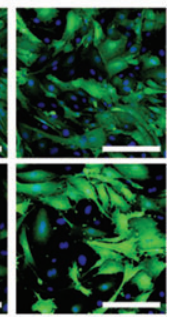

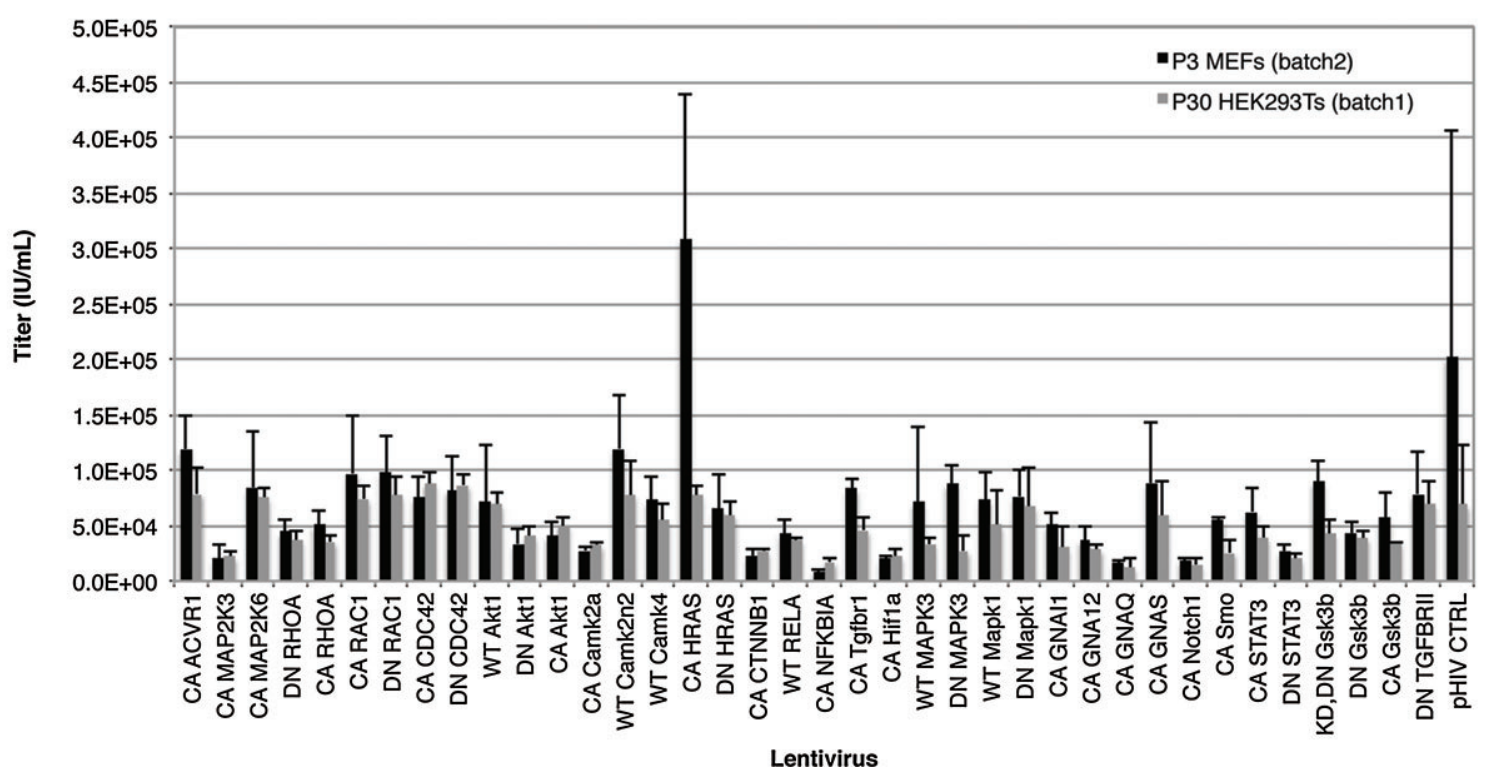

Figure 1. (A) Schematic of the lentiviral vectors used for the reprogramming study. pHIV IG loxP is a self-inactivating lentiviral vector in which a signal transduction gene and GFP are transcribed from an EF1 $\alpha$ promoter. pHIV CTRL is an empty vector used as an infection control. (B) Example images from titering the control virus and a virus containing a constitutively-active ACVR1 on MEF cells. GFP signal was amplified through immunostaining. Nuclei were stained with DAPI. Scale bars are $200 \mu \mathrm{m}$. (C) Titers from two batches for signal transduction gene packaged into lentivirus. Cells were assayed for GFP expression $72 \mathrm{~h}$ post-infection by high-throughput imaging and analysis. Error bars represent $95 \%$ confidence interval. 
Table I. Signal transduction genes used in the study.

\begin{tabular}{|c|c|c|c|c|c|}
\hline \multicolumn{2}{|c|}{ Gene symbol } & \multirow{2}{*}{$\begin{array}{l}\text { Common name } \\
\text { ALK2 }\end{array}$} & \multirow{2}{*}{$\begin{array}{c}\text { Accession } \\
\text { NP_001096 }\end{array}$} & \multirow{2}{*}{$\begin{array}{l}\text { Species } \\
\text { Human }\end{array}$} & \multirow[t]{2}{*}{ Modifications } \\
\hline $\mathrm{CA}$ & ACVR1 & & & & \\
\hline $\mathrm{CA}$ & MAP2K3 & MEK3 & NP_002747 & Human & S189E, T193E, FLAG N-term (E312K mutation) \\
\hline $\mathrm{CA}$ & MAР2К6 & MEK6 & NP_002749 & Human & S207E, T211E, FLAG N-term \\
\hline DN & RHOA & RhoA & NP_001655 & Human & T19N, Myc epitope N-terminus \\
\hline $\mathrm{CA}$ & RHOA & RhoA & NP_001655 & Human & Q63L, Myc epitope N-terminus \\
\hline $\mathrm{CA}$ & RACl & Racl & NP_008839 & Human & Q61L \\
\hline $\mathrm{DN}$ & $R A C 1$ & Rac1 & NP_008839 & Human & $\mathrm{T} 17 \mathrm{~N}$ \\
\hline $\mathrm{CA}$ & $C D C 42$ & CDC42 & NP_001782 & Human & Q61L \\
\hline $\mathrm{DN}$ & $C D C 42$ & $\mathrm{CDC} 42$ & NP_001782 & Human & $\mathrm{T} 17 \mathrm{~N}$ \\
\hline WT & Akt1 & Akt1 & NP_033782 & Mouse & \\
\hline DN & Akt1 & Aktl & NP_033782 & Mouse & T308A, S473A, K179A \\
\hline $\mathrm{CA}$ & Akt1 & Akt1 & NP_033782 & Mouse & T308D, S473D \\
\hline $\mathrm{CA}$ & Camk2a & CaMKII & NP_037052 & Rat & $\mathrm{T} 286 \mathrm{D}$ \\
\hline WT & Camk2n2 & CaMKIIN & NP_067710 & Rat & \\
\hline WT & Camk4 & CaMKIV & NP_036859 & Rat & \\
\hline $\mathrm{CA}$ & HRAS & H-Ras & NP_005334 & Human & $\mathrm{G} 12 \mathrm{~V}$ \\
\hline $\mathrm{DN}$ & HRAS & H-Ras & NP_005334 & Human & S17N \\
\hline $\mathrm{CA}$ & CTNNB1 & $\beta$-Catenin & NP_001895 & Human & S374G, 2xFLAG C-terminus \\
\hline WT & RELA & RelA & NP_068810 & Human & \\
\hline $\mathrm{CA}$ & NFKBIA & ІкB $\alpha$ & NP_065390 & Human & S32A, S36A \\
\hline $\mathrm{CA}$ & Tgfbr1 & ALK5 & P80204 & Rat & GSM after N148, T202D, FLAG N-terminus \\
\hline $\mathrm{CA}$ & Hifla & HIF $1 \alpha$ & Q61221 & Mouse & M1-L400, Q614-STOP(837) \\
\hline WT & MAPK3 & ERK1 & NP_002737 & Human & HA tag N-terminus \\
\hline $\mathrm{DN}$ & MAPK3 & ERK1 & NP_002737 & Human & K71R, HA tag N-terminus \\
\hline WT & Mapk1 & ERK2 & NP_446294 & Rat & \\
\hline $\mathrm{DN}$ & Mapk1 & ERK2 & NP_446294 & Rat & $\mathrm{K} 52 \mathrm{R}$ \\
\hline $\mathrm{CA}$ & GNAI1 & Goil & NP_002060 & Human & Q204L \\
\hline $\mathrm{CA}$ & GNA12 & $\mathrm{G} \alpha 12$ & NP_031379 & Human & Q231L \\
\hline $\mathrm{CA}$ & GNAQ & $\mathrm{G} \alpha \mathrm{q}$ & NP_002063 & Human & Q209L \\
\hline $\mathrm{CA}$ & GNAS & Gos & NP_000507 & Human & Q227L \\
\hline $\mathrm{CA}$ & Notch1 & Notch1 & EDL08321 & Mouse & V1744-S2184, 6 Myc epitopes (C-terminus) \\
\hline $\mathrm{CA}$ & Smo & Smo & NP_036939 & Rat & W539L \\
\hline $\mathrm{CA}$ & STAT3 & STAT3 & NP_003141 & Human & A662C, N664C \\
\hline $\mathrm{DN}$ & STAT3 & STAT3 & NP_644805 & Human & M1-K685 \\
\hline $\mathrm{KD}, \mathrm{DN}$ & Gsk3b & Gsk3 $\beta$ & NP_114469 & Rat & K85R \\
\hline $\mathrm{DN}$ & $G s k 3 b$ & Gsk3 $\beta$ & NP_114469 & Rat & R96A \\
\hline $\mathrm{CA}$ & Gsk3b & Gsk3 $\beta$ & NP_114469 & Rat & S9A \\
\hline DN & TGFBR2 & TGF $\beta$-RII & NP_003233 & Human & M1-I219 \\
\hline
\end{tabular}

Abbreviations: CA, constitutively-active; DN, dominant-negative; KD, kinase-dead; WT, wild-type.

HEK293Ts, presumably due to differences in cell shape/ volume or promoter strength, and immunocytochemistry was necessary to amplify GFP expression for high-content imaging. The infectious viral titers were similar between cell lines and batches (with the exception of constitutively-active HRAS, whose calculated titer was higher on MEF cells than HEK293T cells). Given the similarity in titers, 293T titers were used in reprogramming experiments.

Similar to the quantification of viral titer for viruses encoding signal transduction genes, the cassette virus expressing only Oct4, Sox2, Klf4, and c-Myc, STEMCCA-loxP (Sommer et al., 2010), was titered by immunocytochemistry to quantify OCT4 expression in HEK293T and MEF cells, as the transgene cassette size was close to the lentiviral vector packaging limit and thus precluded the addition of a fluorescent reporter gene (Fig. S1). However, unlike the viruses encoding signaling factors, the STEMCCA titer was much lower (approximately 7.5-fold) on MEF cells than HEK293Ts $\left(6.9 \times 10^{4}\right.$ versus $5.2 \times 10^{5}$ infectious units $/ \mathrm{mL}$, respectively). The viral titer for STEMCCA-loxP on MEFs was thus used in subsequent experiments.

To investigate each gene's effect on reprogramming, 7,500 MEF cells at passage three were plated into 48 well plates. To equalize vector dosages based on the measured titers, virus was added to the supernatant at a multiplicity of infection (MOI) of one (resulting in approximately $63 \%$ of cells infected) for the signaling factors. As the sole exception, cells were infected with vector encoding constitutively-active GNAQ, which had the lowest viral titer, at an MOI of 0.83 (56\% of cells infected). In all cases, cells were infected with the STEMCCA virus at a MOI of $0.13(\sim 12 \%$ of cells infected).

Forty-eight hours post-infection, each well was split into triplicate and plated on mitomycin c-treated MEF feeder layers in mouse embryonic stem cell conditions. Nine days post-infection, the cells were fixed and stained for alkaline phosphatase activity, an early marker of reprogramming (Brambrink et al., 2008). Utilizing the high content 
ImageXpress Micro Screening System (Molecular Devices), $71 \%$ of each well was imaged and analyzed for alkaline phosphatase positive colonies (Fig. 2C). We created customizable analysis software that allowed for the identification of colonies based on a minimum threshold of alkaline phosphatase expression and threshold of colony area. Additionally, this analysis measured individual colony metrics, such as colony area, intensity ranges, and shape factor (Fig. 2B). Example images from the positive control well infected with the control virus (pHIV CTRL) and wells infected with constitutively-active GNAQ or dominantnegative Mapk1 are presented in Figure 2C.

Numbers of alkaline phosphatase positive colonies for each condition are shown in Figure 3. While an exact efficiencyi.e., the fraction of cells infected on day 0 that subsequently underwent reprogramming-cannot be calculated due to conceivable differences in cell division rates among cells expressing different transgenes, an increase in colony number likely corresponds to an increase in reprogramming efficiency. In particular, constitutively-active MAP2K3, constitutively-active NFKBIA, constitutively-active Hifla, dominant-negative Mapk1, constitutively-active GNAI1, and constitutively-active Smo exhibited a significant $(P<0.05)$ increase in colony number compared to the control virus sample. Three of these signaling pathways have been previously implicated in reprogramming biology. First, PD 0325901, a small molecule inhibitor of MAP2K1 and MAP2K2, has been found to help stabilize the pluripotent state when added with a GSK3 $\beta$ inhibitor (Silva et al., 2008). MAP2K1 and MAP2K2 are activators of MAPK1, and as shown in Figure 3, inhibition of Mapk1 increased the colony number. Inhibition of MAPK1 and inhibition of MAP2K1 and MAP2K2 during reprogramming thus presumably act similarly by preventing differentiation (Ying et al., 2008).

Second, hypoxia has been previously found to increase reprogramming efficiency (Yoshida et al., 2009). Analogously,
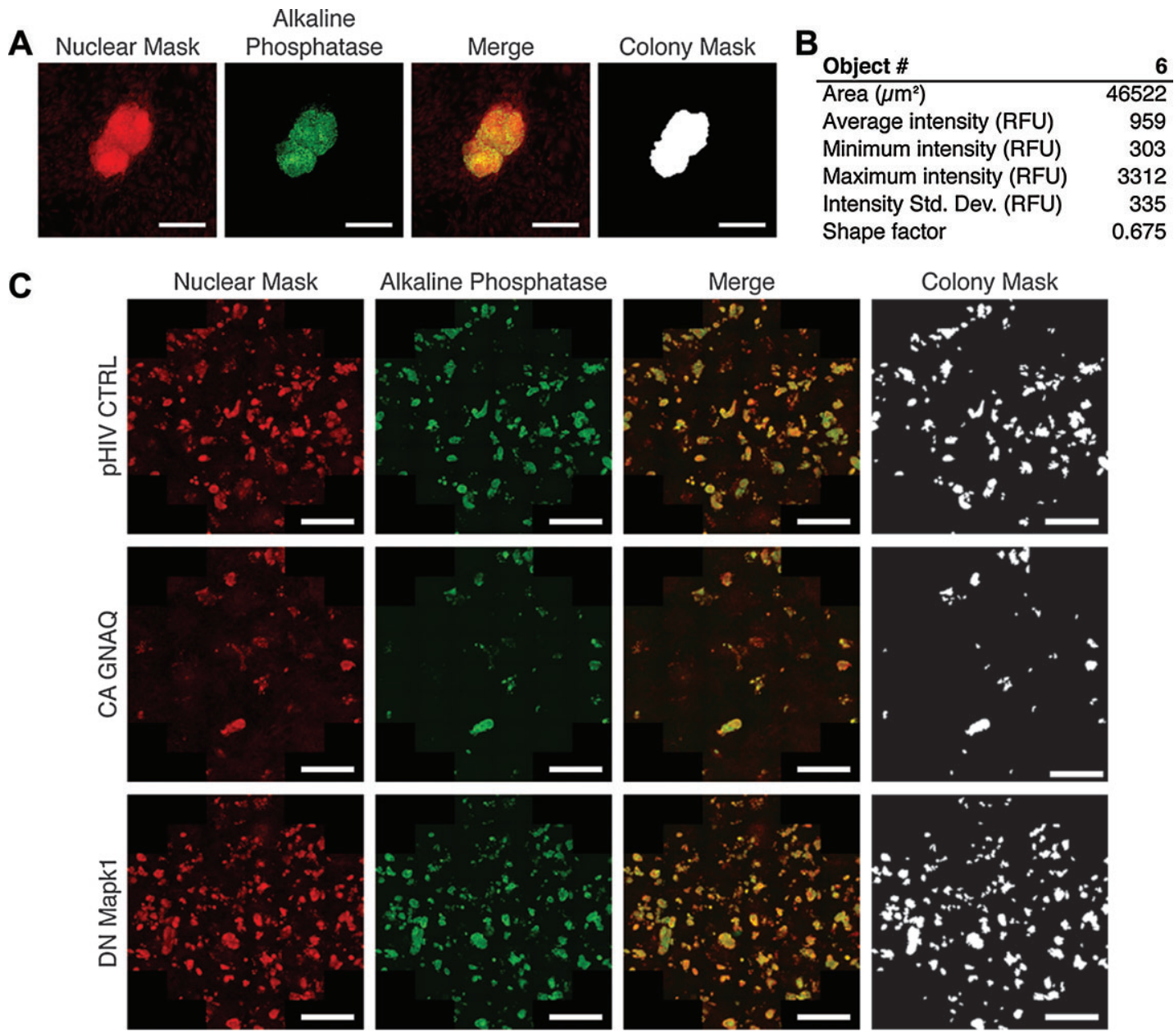

Figure 2. (A) Example of a stained colony from the wild-type Akt1 condition. The nucleus was stained with HCS Nuclear Mask red stain. The alkaline phosphatase was stained with a fluorescent enzymatic-based kit (ATCC). The images are shown individually and merged, and the resultant computer-generated mask from the analysis software from which the area and intensity metrics are measured is shown. Scale bars are $200 \mu \mathrm{m}$. (B) Example of individual colony metrics from the analysis. (C) Three wells are shown: the control pHIV CTRL condition, the constitutively-active GNAQ condition, and the dominant-negative Mapk1 condition. Constitutively-active GNAQ and dominant-negative Mapk1 represent extreme changes in reprogramming efficiency. The minimum colony size measured in the colony mask was $15,000 \mu \mathrm{m}^{2}$. Scale bars are $3 \mathrm{~mm}$. 


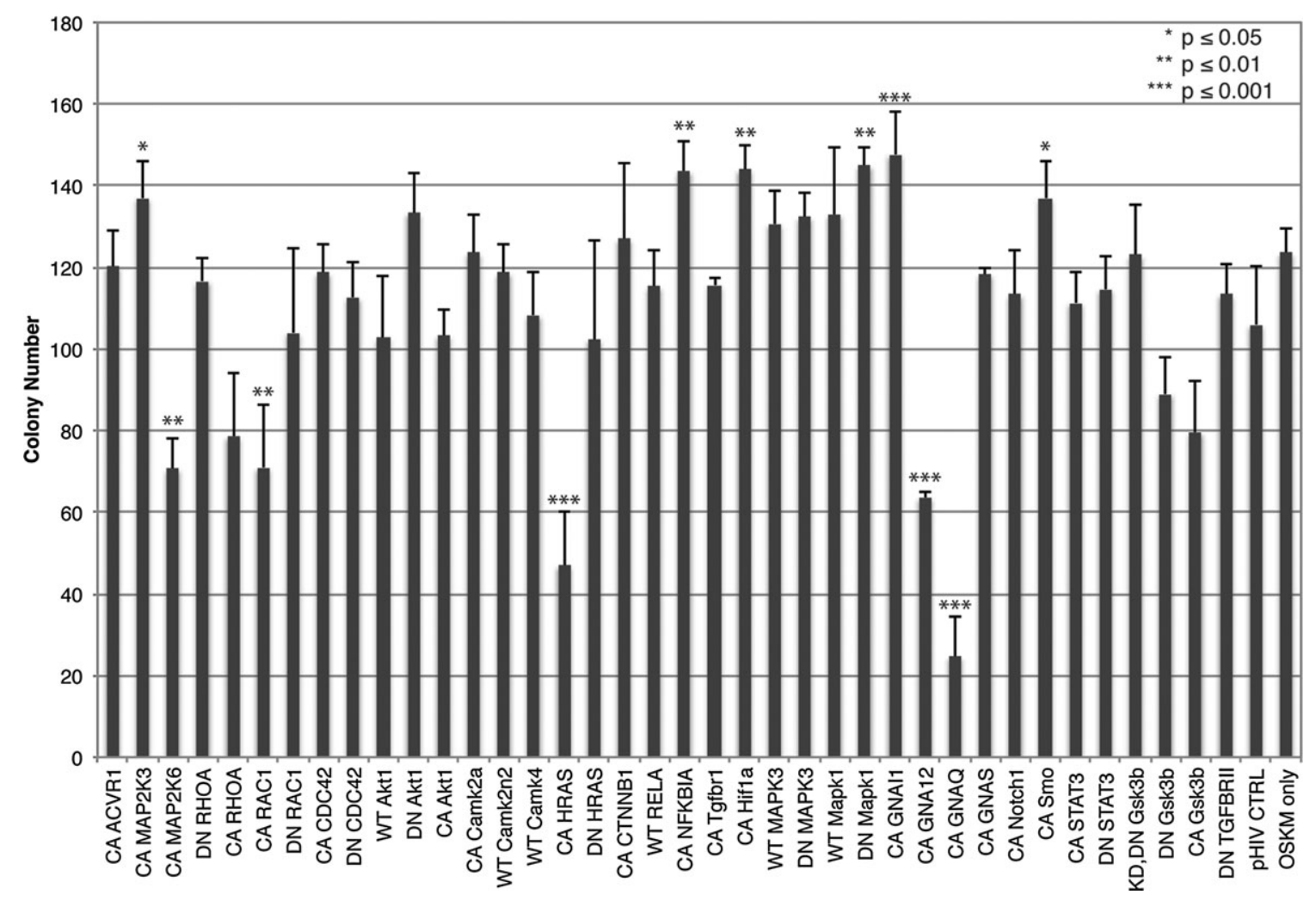

Figure 3. Number of alkaline phosphatase positive colonies 9 days post-infection with a signal transduction gene and $0 c t 4$, Sox2, KIf4, and $c-M y c$ as determined by highcontent imaging and analysis. Statistical significance was measured using ANOVA with a post-hoc Dunnett test relative to the infection control, pHIV CTRL. The minimum colony size measured was $15,000 \mu \mathrm{m}^{2}$. Error bars represent standard deviation.

a constitutively-active Hifla, which mimics a hypoxic environment, increased the number of alkaline phosphatase colonies (Fig. 3). Third, soluble $\mathrm{SHH}$ in combination with OCT4 was able to induce pluripotent stem cells by enhancing an intermediate neural stem cell (NSC)-like state (Moon et al., 2011). While it is unclear whether MEF reprogramming would similarly proceed through a NSC state, in our study a constitutively-active Smo, which activates the $\mathrm{SHH}$ signaling pathway, similarly increased the number of alkaline phosphatase colonies (Fig. 3).

Figure 3 also highlights signaling pathways that are detrimental to reprogramming. Constitutively-active versions of MAP2K6, RAC1, HRAS, GNA12, and GNAQ all led to significant reductions in alkaline phosphatase colonies. While none of the factors has been directly implicated in reprogramming, their signaling pathways offer interesting implications for reprogramming mechanisms.

The STEMCCA loxP vector has been previously utilized for reprogramming (Sommer et al., 2010; Sommer et al., 2009), and we further characterized the effect of signaling factors on such OSKM reprogramming. First, we confirmed that signaling factors that promoted alkaline phosphatase colony formation subsequently led to expression of late-stage markers SSEA1 and NANOG. Specifically, CA $M A P 2 K 3$, CA GNAI1, and CA Smo - which increased the number of colonies-were introduced in combination with OSKM, and cells were assayed for pluripotency marker expression by immunofluorescence nine days post-infection. As shown in Figure S2, the cells infected with a given signaling factor and OSKM had similar SSEA1 and NANOG expression levels and localization compared to the positive control infected with OSKM plus the empty control vector pHIV CTRL.

Second, we quantified gene expression levels for three classes of genes-reprogramming-associated endogenous genes, pluripotency-related genes, and mesenchymal genesin reprogramming conditions with signaling factors. We used quantitative PCR to measure endogenous mRNA transcript levels nine days post-infection in selected conditions that either increased (CA MAP2K3, CA Hifla, CA GNAI1, CA Smo) or decreased colony number (CA MAP2K6 and CA GNAQ) (Fig. 4, S3 normalized to the positive control or negative control, respectively).

First, we quantified endogenous expression of the pluripotency factors Oct4, Nanog, and Sox2, which become transcriptionally-activated during reprogramming. For the conditions that increased colony number (CA MAP2K3, CA Hifla, CA GNAI1, CA Smo), we found similar endogenous gene expression levels with or without signaling factor infection, with few exceptions (CA MAP2K3 and CA Smo had slightly 
A

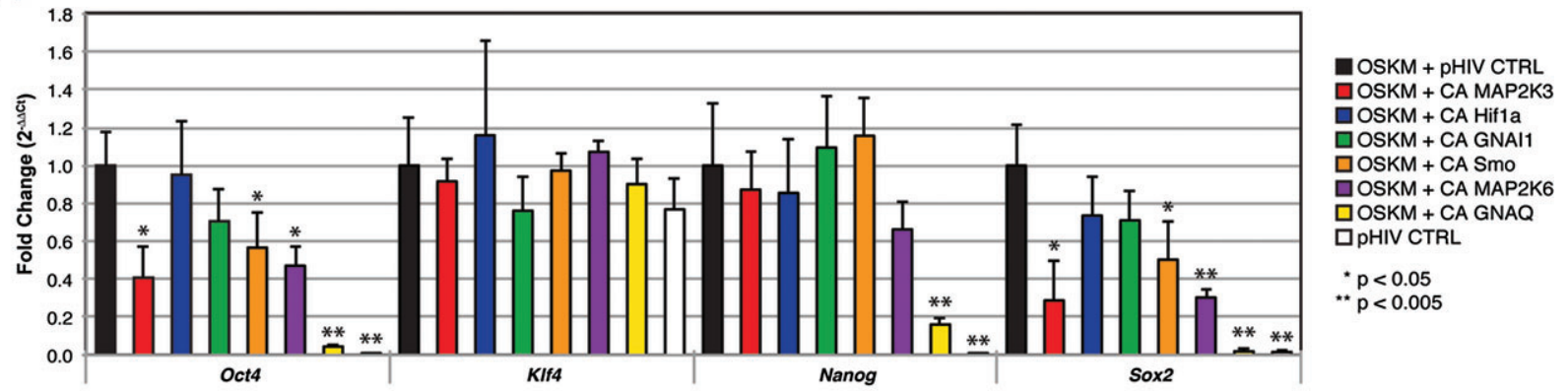

B

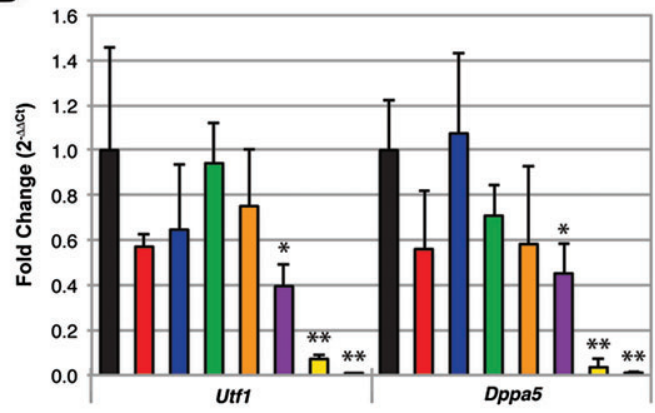

C

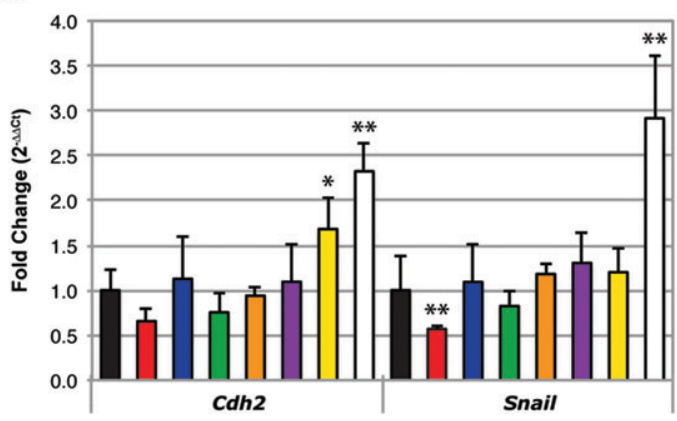

Figure 4. Relative mRNA levels 9 days post-infection with a signal transduction gene and Oct4, Sox2, KIf4, and $c-M y c$ as determined by quantitative PCR. The CA MAP2K3, CA Hif1a, CA GNAI1, and CA Smo conditions represent signaling factors that enhanced reprogramming. The CA MAP2K6 and CA GNAQ conditions represent signaling factors that inhibited reprogramming. The relative mRNA levels were quantified for (A) reprogramming-associated endogenous genes, $(B)$ pluripotency-related genes, or (C) mesenchymal genes. Each gene expression was normalized to the internal control gene, Hprt. Each condition was subsequently normalized to the $0 S K M$ condition. Error bars represent s.d. ( $n=2-$ 3 , biological). Statistical significance was measured using a Students t-Test (two-tailed, homoscedastic) with * $P<0.05$ and ${ }^{* *} P<0.005$. This data is also normalized to the negative control in Figure S3.

reduced Oct4 and Sox2 expression levels relative to OSKM alone) (Fig. 4). Conversely, as expected the potential reprogramming repressors CA MAP2K6 and CA GNAQ, as well as the pHIV CTRL negative control, had significantly lower Oct4, Nanog, and Sox2 gene expression compared to the pHIV CTRL and OSKM positive control with one exception (CA MAP2K6 did not have reduced Nanog expression levels) (Fig. 4).

Second, we studied the pluripotency-associated genes Utf1 and Dppa5, which are also transcriptionally-activated during reprogramming (Kaji et al., 2009). The signaling factor conditions that increased colony number subsequently led to significantly increased $(P<0.005)$ Utf1 and Dppa5 gene expression (Fig. S3B), and there were no significantly differences compared to the positive control (pHIV CTRL and OSKM, Fig. 4B). Finally, the signaling factor conditions that decreased colony number exhibited a significant decrease in pluripotency gene expression compared to the positive control for all cases (Fig. 4B).

Third, we studied the mesenchymal genes Cdh2 and Snail, which are transcriptionally repressed during reprogramming. The signaling factor conditions that increased colony number resulted in a significant reduction $(P<0.05)$ in $C d h 2$ and Snail expression compared to the negative reprogramming control (Fig. S3C). In addition, compared to the positive control
(OSKM and pHIV CTRL infected cells), the potential reprogramming repressor CA GNAQ resulted in significantly higher $C d h 2$ expression $(P<0.05)$ (Fig. 4C). Interestingly for all signaling factor conditions, there was no significant change in Snail expression compared to the positive OSKM control with one exception-CA MAP2K3 had lower Snail expression (Fig. 4C). These results indicate that early alkaline phosphatase expression in response to key signaling factor expression is a valid indicator of later pluripotency gene expression.

These initial results were based on a minimum colony size of $15,000 \mathrm{~mm}^{2}$. However, the custom module for the highthroughput imaging analysis readily allows for changes in the colony size parameter. Figure S4 shows the resultant number of colonies for a smaller size $\left(8,000 \mu \mathrm{m}^{2}\right)$ or a larger size $\left(25,000 \mu \mathrm{m}^{2}\right)$ cutoff. Within the $15,000 \mu \mathrm{m}^{2}$ analysis discussed above, 11 signal transduction factors significantly $(P<0.05)$ altered the number of colonies. However, additional signaling factors were found to significantly modify reprogramming in both the 8,000 and $25,000 \mu^{2}$ cutoff analysis (13 and 12 genes, respectively). For instance, dominant-negative $A k t 1$ was found to increase reprogramming efficiency in both the 8,000 and $25,000 \mu \mathrm{m}^{2}$ analysis but was not statistically significant $(P<0.05)$ in the $15,000 \mu^{2}$ analysis. Additionally, in the $25,000 \mu \mathrm{m}^{2}$ analysis, constitutively-active Gsk $3 b$ was 
found to be significantly $(P<0.01)$ deleterious to reprogramming when considering larger colony sizes, which may act via a previously described mechanism (Ying et al., 2008).

In addition to quantifying the number of colonies after reprogramming, the custom module also measures the total area of all colonies (Fig. S5, or Fig. S6 for smaller $8,000 \mu \mathrm{m}^{2}$ or larger $25,000 \mu \mathrm{m}^{2}$ size cutoffs), which in the hypothetical event that individual colonies were difficult to define and segment could serve as a more effective metric for reprogramming. There were three important differences between the colony number and colony area analysis. First, unlike the $15,000 \mu \mathrm{m}^{2}$ cutoff colony number analysis, for the total area analysis constitutively-active Gsk3ß, which decreased colony area, and constitutively-active CTNNB1, which increased colony area, were statistically significant and further highlighted the importance of the Wnt- $\beta$-catenin pathway in reprogramming. Second, both constitutivelyactive RHOA and RAC1 significantly decreased colony area, which may further elucidate signaling mechanisms detrimental to reprogramming. Third, constitutively-active ACVR1 caused a significant increase in colony area. ACVR1, an important protein in osteogenesis, reportedly yields alkaline phosphatase positive, bone-like cells (Aoki et al., 2001) (Fig. S7). The observed cells-which were large and wellspread-are distinct from the hallmark ES cell colonies of high nucleus to volume ratio cells are thus readily distinguishable as false-positives. In summary, co-expression of signaling factors along with OSKM, coupled with highcontent imaging analysis, enables rapid investigation of how numerous cellular signaling pathways impact the early stages of pluripotency reprogramming.

\section{Discussion}

We have developed a medium-throughput system to investigate the likely complex interactions between signal transduction pathways and early reprogramming mechanisms. In this system, we upregulated and/or downregulated numerous signaling pathways and analyzed their effects on reprogramming using high-content imaging and analysis. In particular, we inserted 38 signaling factors into individual lentiviral vectors, packaged small volumes of each virus, banked frozen viral supernatant, and used high-content imaging to titer lentiviruses and thereby normalize among different viral packaging efficiencies.

By utilizing a robust enzymatic stain with little-to-no background signal for the early reprogramming marker alkaline phosphatase, we generated a quantitative and highthroughput system to assay reprogramming colonies, which are otherwise often counted by hand. The software is capable of measuring numerous population metrics, including final colony number and total area of colonies, as well as individual colony area and a shape factor. These data may yield future insights into the uniformity and distribution of a population of colonies. For instance, a condition with larger colonies compared to the control may be a result of increased reprogramming kinetics or a growth advantage. Additionally, the shape factor may be an indicator of the stability or stochasticity of reprogramming.

By evaluating the factors that increased the number of alkaline phosphatase positive colonies, we found several pathways that were previously known to affect reprogramming. MAPK1, Hifla, and Smo had a significant effect on colony number and reprogramming in this study, consistent with prior work (Moon et al., 2011; Silva et al., 2008; Yoshida et al., 2009). Furthermore, when we analyzed the total area of alkaline phosphatase positive colonies, Wnt- $\beta$-catenin pathway, which has been previously implied in mouse embryonic stem cell biology (Hao et al., 2006) and reprogramming (Silva et al., 2008), was found to alter the area of reprogramming. Specifically elevating $\beta$-catenin signaling with a constitutively-active CTNNB1 resulted in increased colony area, and conversely decreasing the signaling, with a constitutively-active $G s k 3 \beta$, reduced colony area and reprogramming efficiency.

However, we found that while activation of the AKT pathway did not alter reprogramming, a dominant-negative Akt1 increased colony number. This result is contrary to previously published studies showing that AKT binds and positively regulates SOX2 to reprogram cells, and that an inhibitor of PTEN, an AKT pathway antagonist, improves reprogramming efficiency (Jeong et al., 2010; Liao et al., 2013). That said, a small molecule inhibitor of PI3K and thus of the AKT pathway has previously been found to increase reprogramming efficiency (Chen et al., 2011).

Constitutively active versions of $M A P 2 K 3, R A C 1, H R A S$, GNA12, and GNAQ, which yielded fewer alkaline phosphatase positive colonies and were thus detrimental to reprogramming, have interesting implications for reprogramming biology. First, HRAS combined with KLF4 can induce oncogenic transformation of MEFs (Rowland et al., 2005), which here could counteract pluripotency reprogramming; however, this possibility is unlikely since constitutively-active HRAS expression did not induce any apparent expansion of alkaline phosphatase negative colonies (Fig. S8). Alternatively, constitutively-active HRAS has been shown to induce premature senescence in mouse embryonic fibroblasts (Serrano et al., 1997), and HRAS may thus block reprogramming by initiating senescence (Ichida et al., 2009). Finally, constitutively-active RAS, with RAC1 as a possible effector, is important in the epithelial to mesenchymal transition (Edme et al., 2002), which may run counter to the mesenchymal to epithelial transition that is a necessary early event in reprogramming ( $\mathrm{Li}$ et al., 2010).

In addition, GNAQ and MAP2K3 may act though similar mechanisms to suppress reprogramming. GNAQ signals via MAP2K3 and MAP2K6, which ultimately activate MAPK14 (also known as p38 MAPK) (Yamauchi et al., 2001). GNAQ activation of MAP2K3 is independent of RHOA, whereas its activation of MAP2K6 occurs in a RHOA-dependent manner (Yamauchi et al., 2001). Thus, constitutively-active GNAQ, RHOA, and MAP2K6, which each decreased reprogramming efficiency, may potentially act via the same pathway. Conversely, constitutively-active MAP2K3 increased the 
number of alkaline phosphatase colonies, which indicates MAP2K3 may be acting though an alternative mechanism to the GNAQ signaling pathway. Lastly, constitutively-active GNA12-which resulted in significantly fewer alkaline phosphatase colonies in our system-has been shown to upregulate expression of the tumor suppressor p53 (Kim et al., 2007), shown to be a barrier to reprogramming (Hong et al., 2009; Kawamura et al., 2009).

Ultimately, the results of this study may further our understanding of signaling and reprogramming. For instance, our screen has revealed that reprogramming is dramatically impacted by several GTPases, whose role in induced pluripotency has not previously been studied. Not only did we discover activating small GTPases (HRAS, RHOA, RAC1) reduced OCT4, SOX2, KLF4, and C-MYC mediated reprogramming, but activating alpha subunits of heterotrimeric GTPases (GNAI1, GNA12, GNAQ) also had significant impacts in reprogramming, wherein GNA12 and GNAQ decreased and GNAI1 increased efficiency.

In summary, we have created a medium-throughput, quantitative system to modulate major signaling pathways and analyze effects on the early stages of the reprogramming process. These findings can be further explored in future studies to determine their effect on late stage reprogramming in addition to elucidating the mechanistic roles of key canonical signaling pathways in induced pluripotency or in direct lineage reprogramming. In addition, this methodology could readily be implemented to determine whether these signaling pathways could substitute for individual Yamanaka factors. This would benefit reprogramming efforts, since Klf4 and $c-M y c$ are known oncogenes, and even OCT4 expression has been linked with tumor dedifferentiation and progression to a cancer stem cell phenotype (Kumar et al., 2012). Therefore, replacing genes with small molecule or growth factor modulators of signaling could in general enhance the safety of pluripotency or lineage reprogramming. Finally, this lentiviral vector resource can be harnessed to study other roles of signal transduction in mammalian cell and developmental biology, such as stem cell differentiation or lineage reprogramming from one differentiated cell state to another.

\section{Methods}

\section{Cell Culture and Plasmid Construction}

HEK293T cells were maintained in IMDM with 10\% FBS and $1 \%$ penicillin/streptomycin. 129 MEF cells were a kind gift of Lin $\mathrm{He}$ (University of California, Berkeley), isolated as previously described (McCurrach and Lowe, 2001). MEFs were maintained in DMEM (high glucose), 10\% FBS, 1\% GlutaMAX, and 1\% penicillin/streptomycin (Invitrogen). Cells undergoing reprogramming were maintained in mouse embryonic stem cell conditions: DMEM (high glucose), 15\% FBS (Hyclone, SH3007003E), 0.5\% penicillin/streptomycin, $1 \%$ GlutaMAX, $1 \%$ Sodium Pyruvate, 1\% MEM NEAA, $0.1 \mathrm{mM} 2$-mercaptoethanol (Invitrogen), and $1000 \mathrm{U} / \mathrm{mL}$ LIF
(Millipore, ESG1106). Cells were maintained at $37^{\circ} \mathrm{C}$ with $5 \% \mathrm{CO}_{2}$.

Plasmids were obtained from several sources. STEMCCAloxP was a kind gift of Gustavo Mostoslavsky (Boston University) (Sommer et al., 2010). The following plasmids were obtained from Addgene: pHIV EGFP (plasmid 21373) (Welm et al., 2008), pCMV5 ALK-2 Q207D (plasmid 11740) (Macias-Silva et al., 1998), pRC/RSV Flag MKK3(glu) (plasmid 14670) (Raingeaud et al., 1996), and pCDNA3Flag MKK6(glu) (plasmid 13518) (Raingeaud et al., 1996).

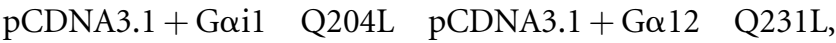

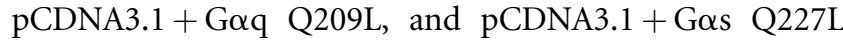
were obtained from Missouri S\&T cDNA Resource Center (www.cdna.org). CLGPIT myc DN RhoA T19N, CLGPIT myc CA RhoA Q63L, CLGPIT CA Rac1(Q61L), CLGPIT DN Rac1 (T17N), CLGPIT CA Cdc42(Q61L), CLGPIT DN Cdc42 (T17N), pBS SK SP Smo, pEN RelA, CLGPIT Akt1, and CLGPIT Akt AAA were described previously (Keung et al., 2011; Lai et al., 2003; Miller-Jensen et al., 2012; Peltier et al., 2007). CLPIT GSK3 $\beta$ K85R, CLPIT GSK3 $\beta$ R96A, and CLPIT GSK3 $\beta$ S9A, cloned from rat cDNA and mutagenized, were kind gifts from Smita Agrawal. MIG STAT3 and MIG DN STAT3 were kind gifts from Il-Hoan $\mathrm{Oh}$ (Oh and Eaves, 2002). pCS2-ICV-6MT mNICD was a kind gift of Raphael Kopan (Washington University) (Kopan et al., 1996), and CLPIT mNICD was a kind gift of Smita Agrawal. pEN IkBa, cloned from cDNA, was a kind gift of Kathryn MillerJensen (Yale University). Wild-type constructs containing CaMKII, CaMKIIN, and CaMKIV were a kind gift of Thomas Soderling (Oregon Health \& Science University) (Chang et al., 1998; Wayman et al., 2008), and CLGPIT CaMKII T286D, CLGPIT CaMKIIN, CLGPIT CaMKIV were a kind gift from Joseph Peltier. The RasG12V construct was a kind gift of Paul Khavari (Stanford University) (Reuter and Khavari, 2006), and CLGPIT HRas G12V was a kind gift of Joseph Peltier. CLGPIT Akt DD and CLGPIT HRas S17N, were mutagenized from WT Akt and HRas G12V constructs, respectively, and were kind gifts of Joseph Peltier. CLPIT CTNNB1 was a kind gift from Anand Asthagiri (Northeastern University). pRK5F FLAG TbRI T202D was a kind gift from Rik Derynck (UCSF) (Choy and Derynck, 1998). pCDNA3.1 + HIF $\triangle$ ODD was a kind gift from David Young (UCSF) (Mace et al., 2007). pCEP4 ERK1, pCEP4 ERK1 K71R, 3XFLAG-CMV7 ERK2, and 3XFLAG-CMV7 ERK2 K52R were kind gifts from Melanie Cobb (University of Texas Southwestern Medical Center) (Robbins et al., 1993). Plasmids containing CTNNB1, Hifla, NFKBIA, Smo, and STAT3 required site directed mutagenesis following the QuikChange protocol (Strategene). Using standard cloning techniques, all genes were inserted into the murine leukemia virus (MLV) retroviral vector CLGPIT (Yu and Schaffer, 2006) and subsequently transferred to the lentiviral vector, pHIV IG loxP. pHIV IG loxP was created by insertion of three oligo constructs into the pHIV EGFP backbone (Welm et al., 2008). The oligos encoded the lox66 site, a multiple cloning site, or the lox71 site. DNA was prepared with QIAGEN Plasmid Midi Kit per manufacturer's instructions. 
Plasmid sequences were verified by restriction enzyme digest and sequencing. These proteins were not functionally tested, and we relied on prior extensive studies as a basis for the screen.

\section{Viral Production and Titering}

For the signal transduction vectors, lentiviral supernatant was produced by HEK293T cells in six well plates using calcium phosphate transfection as previously described (Peltier and Schaffer, 2014). Supernatant was collected $48 \mathrm{~h}$ post transfection, aliquoted, and stored at $-80^{\circ} \mathrm{C}$. For the reprogramming cassette, STEMCCA loxP, the lentiviral transfection and centrifugation were performed as previously described (Peltier and Schaffer, 2014; Sommer et al., 2010). STEMCCA loxP is a second-generation lentivirus, and CLPIT Tat-mCherry (Leonard et al., 2008) was thus added during transfection to promote viral genomic mRNA expression. $0.45 \mu \mathrm{m}$ bottle top filters (Thermo Scientific, 09740-28D) were used to filter the virus, and no sucrose layer was used in ultracentrifugation. The concentrated virus was aliquoted and stored at $-80^{\circ} \mathrm{C}$. Aliquots were only thawed once.

To quantify the amount of virus produced, 1,600 MEF or HEK293T cells were plated in black-walled 96 well plates (E $+\mathrm{K}$ Scientific) coated with gelatin or poly-D-lysine, respectively. After the cells attached, virus was added to the media.

Vector-mediated gene expression was analyzed $72 \mathrm{~h}$ post-infection. For the signal transduction viruses, HEK293T cells were incubated with Hoescht stain (Sigma-Aldrich, B2261) to visualize nuclei. MEF cells were fixed in $4 \%$ paraformaldehyde for $15 \mathrm{~min}$. The cells were blocked and permeabilized with 5\% donkey serum with $0.3 \%$ Triton $\mathrm{X}-100$ in PBS. The anti-GFP primary antibody (Invitrogen, A-11122, 1:500 dilution) was incubated overnight at $4^{\circ} \mathrm{C}$. The secondary antibody (Invitrogen, A-21206, 1:250 dilution) was incubated for $2 \mathrm{~h}$ at room temperature. DAPI (Invitrogen, 1:2000 dilution) was used as a nuclear stain. For STEMCCAloxP virus, both cell types were fixed and stained. An antiOct4 antibody (Santa Cruz Biotechnology, sc-5279, 1:100 dilution) and a secondary antibody (Invitrogen, A-21235, 1:250 dilution) were used.

GFP or OCT4 expression was imaged with the Molecular Devices ImageXpress Micro and analyzed with MetaXpress software. Infectious titers were calculated as previously described (Peltier and Schaffer, 2014).

\section{iPS Cell Reprogramming and Analysis}

7,500 passage $3 \mathrm{MEF}$ cells were plated into 48 well plates. One signal transduction virus $(85-500 \mu \mathrm{L})$ and $14.3 \mu \mathrm{L}$ of STEMCCA-loxP virus were added to each well $8 \mathrm{~h}$ after plating. The following day, the media were replaced with fresh MEF media. $48 \mathrm{~h}$ post-infection, each 48 well was split into triplicate onto MEF feeder layers (GlobalStem, GSC-6101M) in mouse embryonic stem cell media. Media were changed every day.

Nine days post-infection, cells were fixed with $4 \%$ paraformaldehyde for $15 \mathrm{~min}$. Alkaline phosphatase expression was assayed using the ELF Phosphatase Detection Kit (ATCC, SCRR-3010) per manufacturer's instructions. (Note that ATCC kit has been discontinued, and we have found the ELF 97 Endogenous Phosphatase Detection Kit (Invitrogen, E-6601) to be an appropriate substitute). HCS NuclearMask Red stain (Invitrogen, H10326, 1:1000 dilution) was used to visualize nuclei.

The 24 well plates were imaged with a Molecular Devices ImageXpress Micro high-throughput imager, where $71 \%$ of each well's surface area was imaged, avoiding the imaging distortion present near the edges of the plates. A custom filter cube (Semrock BrightLine filters, Excitation: FF01-377/5025, Dichroic: FF409-Di03-25 × 36, Emission: FF01-536/4025) was necessary to detect alkaline phosphatase expression. For image analysis, a custom journal was created in the MetaXpress software to merge the images and identify colonies/regions of alkaline phosphatase expression. Significance was measured in Prism software (GraphPad) using ANOVA with post-hoc Dunnett tests.

\section{Quantitative PCR}

7,500 passage $3 \mathrm{MEF}$ cells were plated for each condition, and one day later cells were infected at an MOI of 0.13 infectious particles/cell with the OSKM virus and an MOI of 0.8-1.0 infectious particles/cell with each signaling factor virus. The cells were passaged into triplicate wells ( 24 well plates) and media changes were conducted as described above in iPS cell reprogramming and analysis. Nine days post-infection, RNA was isolated using a RNeasy Micro Kit (Qiagen, 74004) per manufacturer's instructions. RNA was quantified, normalized, and reverse-transcribed using ThermoScript RT-PCR for First-Strand cDNA Synthesis (Invitrogen, 11146) per manufacturer's instructions. The samples were analyzed for gene expression on a BioRad iQ5 with standard curves, water samples, reverse-transcription control, and melt curves. qPCR primers are listed in Table S1. Data were analyzed by the $\Delta \Delta \mathrm{Ct}$ method compared to the Hprt control gene and the negative control (pHIV CTRL) or positive control (OSKM and pHIV CTRL infected condition), respectively. Statistics were calculated as described in the ABI manual, and significance was measured using a Student's t-Test (twotailed, homoscedastic).

\footnotetext{
We would like to thank Albert Keung and Joseph Peltier for molecular biology assistance. Prof. Lin He and Yong Jin Choi (UC Berkeley) kindly provided MEFs. We would also like to thank Prof. Lin He, Yong Jin Choi, Jaclyn Ho, Shawdee Eshghi, and Teppei Yamaguchi for their helpful discussions about reprogramming. We would like to thank Paula Gedraitis (Molecular Devices), who modified the original code for high-throughput automation and readily helped us updated the code throughout the analysis. This work was funded by National Science Foundation Graduate Fellowship (ALF) and NIH R01 ES020903. We also thank the CIRM/QB3 Berkeley Shared Human Embryonic Stem Cell Facility.
} 


\section{References}

Aoki H, Fujii M, Imamura T, Yagi K, Takehara K, Kato M, Miyazono K. 2001. Synergistic effects of different bone morphogenetic protein type I receptors on alkaline phosphatase induction. J Cell Sci 114(8):1483-1489.

Boeuf H, Hauss C, Graeve FD, Baran N, Kedinger C. 1997. Leukemia inhibitory factor-dependent transcriptional activation in embryonic stem cells. J Cell Biol 138(6):1207-1217.

Brambrink T, Foreman R, Welstead GG, Lengner CJ, Wernig M, Suh H, Jaenisch R. 2008. Sequential expression of pluripotency markers during direct reprogramming of mouse somatic cells. Cell Stem Cell 2(2):151159.

Cartwright P, McLean C, Sheppard A, Rivett D, Jones K, Dalton S. 2005. LIF/ STAT3 controls ES cell self-renewal and pluripotency by a Mycdependent mechanism. Development 132(5):885-896.

Chang BH, Mukherii S, Soderling TR. 1998. Characterization of a calmodulin kinase II inhibitor protein in brain. Proc Natl Acad Sci U S A 95(18):10890-10895.

Chen T, Shen L, Yu J, Wan H, Guo A, Chen J, Long Y, Zhao J, Pei G. 2011. Rapamycin and other longevity-promoting compounds enhance the generation of mouse induced pluripotent stem cells. Aging Cell 10(5):908-911.

Choy L, Derynck R. 1998. The type II transforming growth factor (TGF)-beta receptor-interacting protein TRIP-1 acts as a modulator of the TGF-beta response. J Biol Chem 273(47):31455-31462.

Edme N, Downward J, Thiery JP, Boyer B. 2002. Ras induces NBT-II epithelial cell scattering through the coordinate activities of Rac and MAPK pathways. J Cell Sci 115(Pt 12):2591-2601.

Hao J, Li T-G, Qi X, Zhao D-F, Zhao G-Q. 2006. WNT/beta-catenin pathway up-regulates Stat3 and converges on LIF to prevent differentiation of mouse embryonic stem cells. Dev Biol 290(1):81-91.

Hong H, Takahashi K, Ichisaka T, Aoi T, Kanagawa O, Nakagawa M, Okita K, Yamanaka S. 2009. Suppression of induced pluripotent stem cell generation by the p53-p21 pathway. Nature 460(7259):1132-1135.

Ichida JK, Blanchard J, Lam K, Son EY, Chung JE, Egli D, Loh KM, Carter AC, Di Giorgio FP, Koszka K, Huangfu D, Akutsu H, Liu DR, Rubin LL, Eggan K. 2009. A small-molecule inhibitor of tgf-Beta signaling replaces sox2 in reprogramming by inducing nanog. Cell Stem Cell 5(5):491-503.

Jeong CH, Cho YY, Kim MO, Kim SH, Cho EJ, Lee SY, Jeon YJ, Lee KY, Yao K, Keum YS, Bode AM, Dong Z. 2010. Phosphorylation of Sox 2 cooperates in reprogramming to pluripotent stem cells. Stem Cells 28(12):21412150.

Kaji K, Norrby K, Paca A, Mileikovsky M, Mohseni P, Woltjen K. 2009. Virusfree induction of pluripotency and subsequent excision of reprogramming factors. Nature 458(7293):771-775.

Kawamura T, Suzuki J, Wang YV, Menendez S, Morera LB, Raya A, Wahl GM, Belmonte JCI. 2009. Linking the p53 tumour suppressor pathway to somatic cell reprogramming. Nature 460(7259):1140-1144.

Keung AJ, de Juan-Pardo EM, Schaffer DV, Kumar S. 2011. Rho GTPases mediate the mechanosensitive lineage commitment of neural stem cells. Stem Cells 29(11):1886-1897.

Kim MS, Lee SM, Kim WD, Ki SH, Moon A, Lee CH, Kim SG. 2007. G alpha 12/13 basally regulates p53 through Mdm4 expression. Mol Cancer Res 5(5):473-484.

Kopan R, Schroeter EH, Weintraub H, Nye JS. 1996. G alpha 12/13 basally regulates p53 through Mdm4 expression. Mol Cancer Res 93(4):16831688.

Kumar SM, Liu S, Lu H, Zhang H, Zhang PJ, Gimotty PA, Guerra M, Guo W, Xu X. 2012. Acquired cancer stem cell phenotypes through Oct4mediated dedifferentiation. Oncogene 31(47):4898-4911.

Lai K, Kaspar BK, Gage FH, Schaffer DV. 2003. Sonic hedgehog regulates adult neural progenitor proliferation in vitro and in vivo. Nat Neurosci 6(1):21-27.

Leonard JN, Shah PS, Burnett JC, Schaffer DV. 2008. HIV evades RNA interference directed at TAR by an indirect compensatory mechanism. Cell Host Microbe 4(5):484-494.

Li R, Liang J, Ni S, Zhou T, Qing X, Li H, He W, Chen J, Li F, Zhuang Q, Qin B, $\mathrm{Xu}$ J, Li W, Yang J, Gan Y, Qin D, Feng S, Song H, Yang D, Zhang B, Zeng
L, Lai L, Esteban MA, Pei D. 2010. A mesenchymal-to-epithelial transition initiates and is required for the nuclear reprogramming of mouse fibroblasts. Cell Stem Cell 7(1):51-63.

Liao J, Marumoto T, Yamaguchi S, Okano S, Takeda N, Sakamoto C, Kawano H, Nii T, Miyamato S, Nagai Y, Okada M, Inoue H, Kawahara K, Suzuki A, Miura Y, Tani K. 2013. Inhibition of PTEN tumor suppressor promotes the generation of induced pluripotent stem cells. Mol Ther 21(6):1242-1250.

Mace KA, Yu DH, Paydar KZ, Boudreau N, Young DM. 2007. Sustained expression of Hif-1alpha in the diabetic environment promotes angiogenesis and cutaneous wound repair. Wound Repair Regen 15(5):636-645.

Macias-Silva M, Hoodless PA, Tang SJ, Buchwald M, Wrana JL. 1998. Specific activation of Smad1 signaling pathways by the BMP7 type I receptor, ALK2. J Biol Chem 273(40):25628-25636.

Maherali N, Hochedlinger K. 2009. Tgfbeta signal inhibition cooperates in the induction of iPSCs and replaces Sox2 and cMyc. Curr Biol 19(20):1718-1723.

Marson A, Foreman R, Chevalier B, Bilodeau S, Kahn M, Young R, Jaenisch R. 2008. Wnt signaling promotes reprogramming of somatic cells to pluripotency. Cell Stem Cell 3(2):132-135.

Matsuda T, Nakamura T, Nakao K, Arai T, Katsuki M, Heike T, Yokota T. 1999. STAT3 activation is sufficient to maintain an undifferentiated state of mouse embryonic stem cells. EMBO J 18(15):4261-4269.

McCurrach ME, Lowe SW. 2001. Methods for studying pro- and antiapoptotic genes in nonimmortal cells. Methods Cell Biol 66:197227.

Miller-Jensen K, Dey SS, Pham N, Foley JE, Arkin AP, Schaffer DV. 2012. Chromatin accessibility at the HIV LTR promoter sets a threshold for NF-kappaB mediated viral gene expression. Integr Biol (Camb) 4(6):661-671.

Moon JH, Heo JS, Kim JS, Jun EK, Lee JH, Kim A, Kim J, Whang KY, Kang YK, Yeo S, Lim HJ, Han DW, Kim DW, Oh S, Yoon BS, Schöler HR, You S. 2011. Reprogramming fibroblasts into induced pluripotent stem cells with Bmil. Cell Res 21(9):1305-1315.

Niwa H, Burdon T, Chambers I, Smith A. 1998. Self-renewal of pluripotent embryonic stem cells is mediated via activation of STAT3. Genes Dev 12(13):2048-2060.

Oh IH, Eaves CJ. 2002. Overexpression of a dominant negative form of STAT3 selectively impairs hematopoietic stem cell activity. Oncogene 21(31):4778-4787.

Peltier J, O'Neill A, Schaffer DV. 2007. PI3K/Akt and CREB regulate adult neural hippocampal progenitor proliferation and differentiation. Dev Neurobiol 67(10):1348-1361.

Peltier J, Schaffer DV. Viral packaging and transduction of adult hippocampal neural progenitors. Methods Mol Biol 621:103-116.

Raingeaud J, Whitmarsh AJ, Barrett T, Derijard B, Davis RJ. 1996. MKK3and MKK6-regulated gene expression is mediated by the p38 mitogenactivated protein kinase signal transduction pathway. Mol Cell Biol 16(3):1247-1255.

Raz R, Lee CK, Cannizzaro LA, d'Eustachio P, Levy DE. 1999. Essential role of STAT3 for embryonic stem cell pluripotency. Proc Natl Acad Sci U S A 96(6):2846-2851.

Reuter JA, Khavari PA. 2006. Use of conditionally active ras fusion proteins to study epidermal growth, differentiation, and neoplasia. Methods Enzymol 407:691-702.

Robbins DJ, Zhen E, Owaki H, Vanderbilt CA, Ebert D, Geppert TD, Cobb MH. 1993. Regulation and properties of extracellular signal-regulated protein kinases 1 and 2 in vitro. J Biol Chem 268(7):5097-5106.

Rowland BD, Bernards R, Peeper DS. 2005. The KLF4 tumour suppressor is a transcriptional repressor of p53 that acts as a context-dependent oncogene. Nat Cell Biol 7(11):1074-1082.

Samavarchi-Tehrani P, Golipour A, David L, Sung H-K, Beyer TA, Datti A, Woltjen K, Nagy A, Wrana JL. 2010. Functional genomics reveals a BMPdriven mesenchymal-to-epithelial transition in the initiation of somatic cell reprogramming. Cell Stem Cell 7(1):64-77.

Serrano M, Lin AW, McCurrach ME, Beach D, Lowe SW. 1997. Oncogenic ras provokes premature cell senescence associated with accumulation of $\mathrm{p} 53$ and p16INK4a. Cell 88(5):593-602. 
Silva J, Barrandon O, Nichols J, Kawaguchi J, Theunissen TW, Smith A. 2008 Promotion of reprogramming to ground state pluripotency by signal inhibition. PLoS Biol 6(10):e253.

Sommer CA, Sommer AG, Longmire TA, Christodoulou C, Thomas DD, Gostissa M, Alt FW, Murphy GJ, Kotton DN, Mostoslavsky G. 2010. Excision of reprogramming transgenes improves the differentiation potential of iPS cells generated with a single excisable vector. Stem Cells 28(1):64-74.

Sommer CA, Stadtfeld M, Murphy GJ, Hochedlinger K, Kotton DN, Mostoslavsky G. 2009. Induced pluripotent stem cell generation using a single lentiviral stem cell cassette. Stem Cells 27(3):543549 .

Takahashi K, Yamanaka S. 2006. Induction of pluripotent stem cells from mouse embryonic and adult fibroblast cultures by defined factors. Cell 126(4):663-676.

Wayman GA, Lee YS, Tokumitsu H, Silva AJ, Soderling TR. 2008. Calmodulin-kinases: modulators of neuronal development and plasticity. Neuron 59(6):914-931.

Welm BE, Dijkgraaf GJ, Bledau AS, Welm AL, Werb Z. 2008. Lentiviral transduction of mammary stem cells for analysis of gene function during development and cancer. Cell Stem Cell 2(1):90-102.
Yamauchi J, Tsujimoto G, Kaziro Y, Itoh H. 2001. Parallel regulation of mitogen-activated protein kinase kinase 3 (MKK3) and MKK6 in Gqsignaling cascade. J Biol Chem 276(26):23362-23372.

Ying QL, Nichols J, Chambers I, Smith A. 2003. BMP induction of Id proteins suppresses differentiation and sustains embryonic stem cell self-renewal in collaboration with STAT3. Cell 115(3):281-292.

Ying QL, Wray J, Nichols J, Batlle-Morera L, Doble B, Woodgett J, Cohen P, Smith A. 2008. The ground state of embryonic stem cell self-renewal. Nature 453(7194):519-523.

Yoshida Y, Takahashi K, Okita K, Ichisaka T, Yamanaka S. 2009. Hypoxia enhances the generation of induced pluripotent stem cells. Cell Stem Cell 5(3):237-241.

Yu JH, Schaffer DV. 2006. Selection of novel vesicular stomatitis virus glycoprotein variants from a peptide insertion library for enhanced purification of retroviral and lentiviral vectors. J Virol 80(7):3285-3292.

\section{Supporting Information}

Additional Supporting Information may be found in the online version of this article at the publisher's web-site. 